\title{
A new case of SMA phenotype without epilepsy due to biallelic variants in ASAH1
}

\author{
Nadine AME van der Beek $\mathbb{D}^{1} \cdot$ Isabelle Nelson ${ }^{2} \cdot$ Roseline Froissart $^{3} \cdot$ Thierry Levade $^{4} \cdot$ Virginie Garcia $^{5}$. \\ Emmanuelle Lacene ${ }^{6,7,8,9}$. Anne Boland ${ }^{7}$. Cécile Masson ${ }^{7,8}$ - Norma B. Romero ${ }^{6,7,8,9}$ - Tanya Stojkovic ${ }^{9}$. \\ Gisèle Bonne $\mathbb{1}^{2} \cdot$ Anthony Béhin ${ }^{9}$
}

Received: 18 January 2018 / Revised: 3 August 2018 / Accepted: 9 August 2018 / Published online: 5 October 2018

(c) European Society of Human Genetics 2018

Variants in ASAHI are known to be associated with two disease entities, namely: (1) Farber lipogranulomatosis, a rare disease characterized by subcutaneous, periarticular nodules, painful contractures, a hoarse voice, central nervous system involvement, and sometimes hepatosplenomegaly (OMIM \#228000), and: (2) spinal muscular atrophy associated with progressive myoclonic epilepsy (SMA-PME), a childhood-onset, progressive lower motor neuron degeneration with refractory, myoclonic epilepsy (OMIM \#159950). Other features may include hearing loss, tremor, or cognitive dysfunction. Thus far, around 25 individuals with SMA-PME have been reported-though not all cases were confirmed by genotyping and/or documentation of deficient acid ceramidase activity. [1-9] Recently, Filosto et al. [10] described two siblings with an SMA phenotype, but without myoclonic epilepsy due to variants in $A S A H 1$, further expanding the phenotypical spectrum. We now report a third, unrelated, case with ASAH1 related SMA.

A now 24-year-old female with no other relevant past medical history first sought care for slowly progressive

Anthony Béhin

anthony.behin@aphp.fr

1 Department of Neurology, Center for Lysosomal and Metabolic Diseases, Erasmus MC University Medical Center, Rotterdam, The Netherlands

2 Sorbonne Universités, UPMC Univ Paris 06, INSERM UMRS 974, Center of Research in Myology, Institut de Myologie, F75013 Paris, France

3 Service de Biochimie et Biologie Moléculaire Grand Est, Unité Médicale Pathologies Métaboliques, Erythrocytaires et Dépistage Périnatal, Centre de Biologie et de Pathologie Est, Hospices Civils de Lyon, Bron, France

4 Laboratoire de Biochimie Métabolique, Institut Fédératif de Biologie, Hôpital Purpan, and INSERM U1037, CRCT, muscle weakness and muscle cramps in the arms and legs at the age of 19 years. She was born to non-consanguineous parents, and early developmental motor milestones had been achieved at appropriate ages. Her first ambulatory problems-walking more slowly - arose at the age of eight years. She had been an active dancer during her childhood, but experienced problems crouching and maintaining some positions from age 14 on and had to stop practicing at the age of 15 . From this age onward, she increasingly experienced problems with climbing stairs (by now systematically using the banister), raising herself from the floor, and carrying heavy loads above shoulder height. At her most recent visit to our hospital (at 23 years of age), she was still able to walk about two kilometers, but had near-falls 2-3 times a year. Additionally, she complained of mild exercise dyspnea. She did not have any sleep disturbances, or swallowing difficulties. She had never experienced seizures or myoclonic jerks. On examination, she had atrophy and fasciculations of the tongue, postural tremor of the hands (improved by beta-blockers), bilateral scapular winging, axial weakness (neck flexion MRC grade 4), bilateral

Toulouse, France

5 INSERM U1037, CRCT, Toulouse, France

6 APHP, Centre de Référence de Pathologie Neuromusculaire Nord/ Est/Ile-de-France, Institut de Myologie, Laboratoire de Pathologie Risler, GH Pitié-Salpêtrière, Paris, France

7 Centre National de Recherche en Génétique Humaine (CNRGH), Evry, France

8 Bioinformatics Core Facility, Université Paris Descartes Structure Fédérative de Recherche Necker, INSERM US24/ CNRS, UMS3633 Paris, France

9 APHP, Centre de Référence de Pathologie Neuromusculaire Nord/ Est/Ile-de-France, Institut de Myologie, GH Pitié-Salpêtrière, Paris, France 
proximal weakness of the arms (shoulder anteflexion MRC grade 3, shoulder abduction/elbow flexion/elbow extension MRC grade 4) and legs (hip flexors MRC grade 3, knee flexors MRC grade 4), and also a slight distal weakness of the hands. Sensory investigations were normal.

Ancillary laboratory investigations repeatedly showed normal creatine kinase levels. Needle electromyography showed large amplitude $(>10 \mathrm{mV})$ motor unit action potentials (MUPs) without active denervation, while a muscle biopsy showed a strong predominance of type I muscle fibers with fiber type-grouping, both consistent with a chronic neurogenic disease. Muscle MRI showed thinning of the proximal musculature, without major focal atrophy. Cardiac and pulmonary investigations did not reveal abnormalities; abdominal ultrasound showed no hepatosplenomegaly; EEG, ophthalmological examination and hearing were normal; brain MRI was normal, apart from a small cyst of the pineal gland.

Genetic testing for SMN1 (performed twice) did not reveal any deletions, point mutations, or large rearrangements. As a next step, exome sequencing was performed with the informed consent of the patient in the context of the "French Myocapture consortium", which revealed two novel variants in $A S A H 1$ : c. $77 \mathrm{C}>\mathrm{G}$; p.(Pro26Arg) in exon 1, and c. $125+1 \mathrm{G}>\mathrm{A}$ affecting the donor splice site of intron 2 , leading to an abnormal (probably lacking exon 2) and unstable transcript (RefSeq NG_008985.1, NM_177924.3). Both variants were confirmed by Sanger sequencing. Analyses in the family confirmed the bi-parental segregation. Subsequently, acid ceramidase activity was tested in leukocytes, showing a deficient enzymatic activity $(0.89 \mathrm{nmol} /$ $\mathrm{h} / \mathrm{mg}$ protein; control mean value 18.85), strongly advocating for the diagnosis of ASAHI related SMA (www.lovd. nl/asah1 (individual \# 00163649); exons numbered according to NG_008985.1).

Finding the genetic origin of SMA in cases with no SMN1 variants remains difficult. In the case reported here, exome sequencing revealed two novel variants that were expected to cause the disease: the variant c. $77 \mathrm{C}>\mathrm{G}$, p.(Pro26Arg), is located in a relatively conserved region, and is predicted to affect protein function by Polyphen ${ }^{\circledR}$ and Mutation Taster ${ }^{\circledR}$. On the other allele, the variant c.125+1G $>$ A affects a consensus splice site, and variants near this position (i.e., c. $124 \mathrm{~A}>\mathrm{G}$ and $\mathrm{c} .125 \mathrm{C}>\mathrm{T}$ ) have already been implicated in the SMA-epilepsy phenotype $[2,5,10]$. Analysis and sequencing of the cDNA obtained from the patient's cultured lymphoblasts revealed the presence of only the ASAHI transcript carrying the c. $77 \mathrm{C}>\mathrm{G}$ substitution, suggesting that the c. $125+1 \mathrm{G}>\mathrm{A}$ variant leads to an unstable transcript.

Both variants have never been reported in Exome Sequencing Project, 1000 Genomes Project, or Exome Aggregation Consortium. The mode of inheritance in the family was compatible with autosomal recessive transmission.
ASAHI encodes acid ceramidase ( $\mathrm{N}$-acylsphingosine amidohydrolase), a lysosomal enzyme with a heterodimeric structure that plays a major part in the sphingolipid rheostat. This biochemical reaction is crucial for the regulation of apoptosis. In a zebrafish model with morpholino knockdown of $A S A H 1$, a marked loss of motor-neuron axonal branching was noticed, together with an increased apoptosis in the spinal cord [2], which seems compatible with the development of pure SMA in humans. Considering the potential causative role of ASAHI detected variants, the strong decrease in acid ceramidase activity (around $5 \%$ of normal) observed in the patient's leukocytes brought further evidence towards its implication. This confirms the role played by this gene in pure SMA phenotypes as described by Filosto et al. [10] in two sisters from a Pakistani consanguineous family harboring a homozygous c.124A $>\mathrm{G}$ (r.124a $>$ g; (p.Thr42Ala)) variant. Despite an earlier onset, both display a clinical pattern very similar to the one observed in our case.

In conclusion, the finding of variants in two unrelated families supports the recommendation for including ASAHI in gene panels for SMA.

Acknowledgements NAMEvdB received a grant from the Prinses Beatrix Spierfonds. Exome sequencing took place as part of the "Myocapture sequencing project", financed through the France Génomique National infrastructure ("Investissements d'Avenir" program ANR-10-INBS-09) and the Fondation Maladies Rares.

Authors contributions NAMEvdB analysis and interpretation of data, drafting of manuscript. IN acquisition of data, critical revision of manuscript for intellectual content. RF acquisition of data, critical revision of manuscript for intellectual content. TL acquisition of data, critical revision of manuscript for intellectual content. VG acquisition of data, critical revision of manuscript for intellectual content. EL acquisition of data, critical revision of manuscript for intellectual content. $\mathrm{AB}$ acquisition of data, critical revision of manuscript for intellectual content. CM acquisition of data, critical revision of manuscript for intellectual content. NBR acquisition of data, critical revision of manuscript for intellectual content. GB acquisition of data, critical revision of manuscript for intellectual content. $\mathrm{AB}$ acquisition of data, analysis and interpretation of data, critical revision of manuscript for intellectual content. All authors read, revised and approved the final manuscript.

\section{Compliance with ethical standards}

Conflict of interest The authors declare that they have no conflict of interest.

Statement All the raw data presented in this study are available to the corresponding author.

\section{References}

1. Jankovic J, Rivera VM. Hereditary myoclonus and progressive distal muscular atrophy. Ann Neurol. 1979;6:227-31. 
2. Zhou J, Tawk M, Tiziano FD, Veillet J, Bayes M, Nolent F, et al. Spinal muscular atrophy associated with progressive myoclonic epilepsy is caused by mutations in ASAH1. Am J Hum Genet. 2012;91:5-14.

3. Dyment DA, Sell E, Vanstone MR, Smith AC, Garandeau D, Garcia V, et al. Evidence for clinical, genetic and biochemical variability in spinal muscular atrophy with progressive myoclonic epilepsy. Clin Genet. 2014;86:255-563.

4. Gan JJ, Garcia V, Tian J, Tagliati M, Parisi JE, Chung JM, et al. Acid ceramidase deficiency associated with spinal muscular atrophy with progressive myoclonic epilepsy. Neuromuscul Disord. 2015;25:959-63.

5. Rubboli G, Veggiotti P, Pini A, Berardinelli A, Cantalupo G, Bertini E et al. Spinal muscular atrophy associated with progressive myoclonic epilepsy: a rare condition caused by mutations in ASAH1. Epilepsia. 2015;56:692-8.
6. Teoh HL, Solyom A, Schuchman EH, Mowat D, Roscioli T, Farrar M, et al. Polyarticular arthritis and spinal muscular atrophy in acid ceramidase deficiency. Pediatrics. 2016;138:e20161068.

7. Kernohan KD, Frésard L, Zappala Z, Hartley T, Smith KS, Wagner $\mathrm{J}$, et al. Whole-transcriptome sequencing in blood provides a diagnosis of spinal muscular atrophy with progressive myoclonic epilepsy. Hum Mut. 2017;38:611-4.

8. Topaloglu H, Melki J. Spinal muscular atrophy associated with progressive myoclonus epilepsy. Epileptic Disord. 2016;18:S128-34.

9. Schuchman EH. Acid ceramidase and the treatment of ceramide diseases: the expanding role of enzyme replacement therapy. Biochim Biophys Acta. 2016;1862:1459-71.

10. Filosto M, Aureli M, Castelotti B, Rinaldi F, Schiumarini D, Valsecchi M, et al. ASAH1 variant causing a mild SMA phenotype with no myoclonic epilepsy: a clinical, biochemical and molecular study. Eur J Hum Genet. 2016;24:1578-83. 